\title{
Armand Imbert (1850-1922), la science du travail et la paix sociale
}

Armand Imbert (1850-1922), The Science of Work and social Peace

\section{Thomas Le Bianic et François Vatin}

\section{OpenEdition}

\section{Journals}

Édition électronique

URL : http://journals.openedition.org/travailemploi/4615

DOI : $10.4000 /$ travailemploi.4615

ISSN : 1775-416X

Éditeur

DARES - Ministère du Travail

Édition imprimée

Date de publication : 30 juin 2007

Pagination : 7-19

ISSN : 0224-4365

\section{Référence électronique}

Thomas Le Bianic et François Vatin, « Armand Imbert (1850-1922), la science du travail et la paix sociale », Travail et Emploi [En ligne], 111 | juillet-septembre 2007, mis en ligne le 15 septembre 2008, consulté le 22 septembre 2020. URL : http://journals.openedition.org/travailemploi/4615 ; DOI :

https://doi.org/10.4000/travailemploi.4615 


\title{
Armand Imbert (1850-1922), la science du travail et la paix sociale
}

\author{
Thomas Le Bianic (*), François Vatin ${ }^{* *}$ )
}

\begin{abstract}
Armand Imbert fut un précurseur en matière de physiologie et de médecine du travail en France: par une démarche originale, à la fois scientifique et sociale, qui a rencontré l'intérêt des milieux politiques et syndicaux, il a tenté de jeter les bases d'une science du travail qui soit susceptible d'apporter un arbitrage scientifique aux conflits sociaux et d'influer sur les relations professionnelles et le dialogue social. L'originalité de son action en fait un auteur toujours proche des réflexions contemporaines.
\end{abstract}

Une histoire des débuts du ministère du Travail peut difficilement rester silencieuse sur le contexte scientifique qui entoura sa création. Si l'influence des sciences économiques et sociales au sein d'une institution comme l'Office du travail est désormais bien connue ( $c f$. Moret-Lespinet, 1997; Soubiran, 2007), il reste encore à mener une étude systématique des autres disciplines, notamment médicales, qui sont venues instrumenter l'action de l'administration du travail dans les multiples domaines d'intervention qui furent les siens au début du $\mathrm{XX}^{\mathrm{e}}$ siècle : hygiène et sécurité, prévention des accidents du travail, maladies professionnelles, règlement des conflits du travail, etc. Quelques travaux pionniers ont déjà souligné le rôle important de nouvelles disciplines comme l'hygiène industrielle, la bactériologie ou la physiologie dans l'action réglementaire du ministère du Travail, ainsi que dans les pratiques de terrain de ses agents au sein des entreprises (voir notamment RIBEILL, 1980; VIET, 1994; VIET, Ruffat, 1999). Ces disciplines apportent à la jeune administration de nouvelles méthodes d'observation, qui lui permettent de déployer son action législative sur des bases plus objectives, et de légitimer son intervention aux yeux des ouvriers et du monde patronal (1).

Le plus souvent, le ministère s'entoure des compétences de personnalités scientifiques extérieures au ministère, qu'il invite à participer aux comités qu'il met en place (notamment la Commission d'hygiène industrielle, instituée en 1900, ou le Comité consultatif des arts et manufactures, d'origine plus

(*) Université Paris-Dauphine - CERSO. lebianic@dauphine.fr (**) Université de Paris X - IDHE-Nanterre. vatin@u-paris10.fr (1) En témoigne par exemple le caractère souvent très technique des mémoires produits par les inspecteurs du travail dans le Bulletin de l'inspection du travail dans la période 1895-1914.

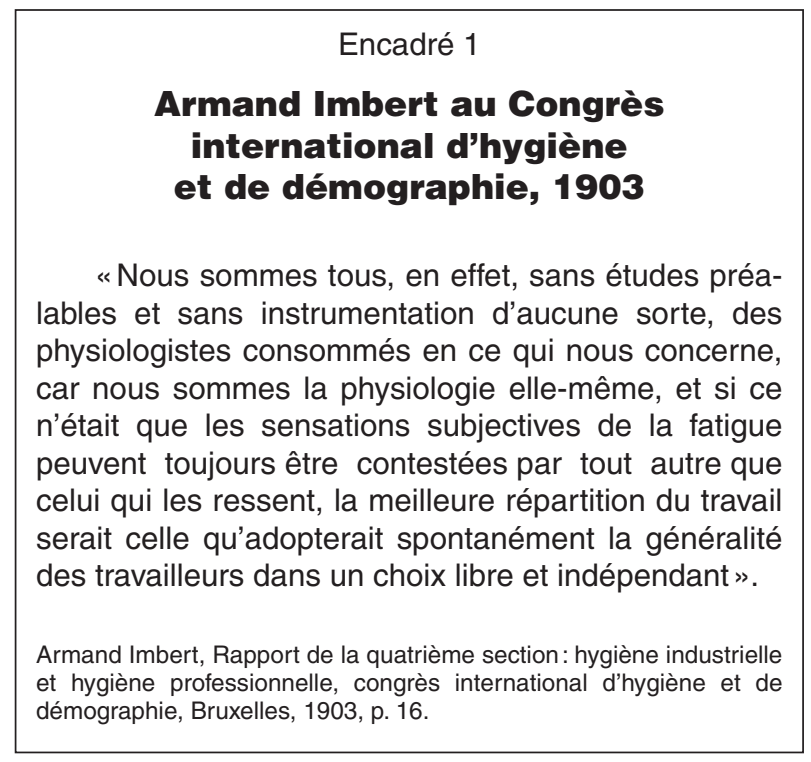

ancienne(2)). Parfois, les relations passent par des contacts plus informels entre administrateurs d'un côté, savants, ingénieurs ou médecins de l'autre. Le ministère du Travail participe aussi à diverses reprises au financement d'enseignements, de chaires ou de laboratoires de recherche au Conservatoire des arts et métiers comme le Cours d'hygiène industrielle d'Heim de Balsac (1905) ou le Laboratoire d'études du travail professionnel de Jules Amar, créé en 1913. S’il semble difficile, à se stade, de se livrer à une étude exhaustive des réseaux scientifiques dont s'entoure le ministère du Travail à ses débuts, nous examinerons en détail les relations qu'il a nouées avec un physiologiste montpelliérain aujourd'hui oublié, Armand Imbert, qui déve-

(2) Ce comité remontait à la création en 1791 du Bureau consultatif des arts et manufactures, chargé de subventionner les inventeurs, et dont les fonctions s'étaient élargi au XIX ${ }^{\mathrm{e}}$ à l'étude de toutes les questions concernant le commerce et l'industrie, notamment celle des établissements incommodes et insalubres. 
loppa dans les années 1890-1920, c’est-à-dire dans les années qui précèdent la création du ministère du Travail en 1906 et celles qui lui succèdent, une conception originale de la science du travail. Celleci est à la fois représentative des prises de position de nombreux savants s'intéressant au travail dans cette période, au carrefour de l'action administrative et de l'expertise scientifique, mais est aussi riche d'enseignement par la grande actualité de la pensée d'Armand Imbert.

Après une rapide biographie de ce personnage, qui permettra notamment de voir comment elle a croisé, à plusieurs reprises, l'histoire du ministère du Travail, nous nous attacherons à présenter sa conception épistémologique originale d'une science du travail professionnel susceptible, à son sens, d'arbitrer les conflits sociaux. Comme nous le verrons, sa démarche, aux antipodes de tout scientisme, car elle fait du travailleur lui-même le meilleur connaisseur de sa physiologie, débouche sur une logique de «relations professionnelles », qui est soutenue, mais non close par la science.

\section{Armand Imbert, médecin, savant et humaniste}

Armand Imbert est né en 1850 à Seyne-les-Alpes (actuelles Alpes-de-Haute-Provence), dans un milieu de la petite bourgeoisie intellectuelle. Son père, issu d'une famille de petits agriculteurs, fut instituteur, puis professeur de mathématiques en collège. Ses trois fils devinrent médecins. Armand était l'aîné. Le cadet, Henri (1864-1928), fut professeur de chimie à l'école de pharmacie de Montpellier de 1902 jusqu'à sa mort. Le benjamin, Léon (1868-1955), chirurgien, fut doyen de la faculté de médecine de Marseille et s'intéressa aux problèmes du travail dans le sillage de son aîné (Léon IMBERT, 1913)(3).

Après un bref passage dans l'enseignement au collège d'Orange (1870) et au lycée de Lyon (1877), Armand Imbert entreprend des études à Lyon, où il obtient un doctorat en physique (IMBERT, 1880) et un doctorat en médecine (IMBERT, 1883). Il est reçu la même année à l'agrégation des facultés de médecine et est affecté, en 1884, comme professeur de physique à l'école de pharmacie de Montpellier. En 1889, il est nommé titulaire de la chaire de physique médicale de la Faculté de médecine de cette même ville, poste qu'il occupa jusqu'à la fin de sa carrière. Sa reconnaissance académique est achevée par son élection comme membre correspondant de l'Académie des sciences en 1899.

(3) Dans cet ouvrage, Léon Imbert rend hommage aux travaux de son frère, qu'il présente comme l'un des précurseurs de la médecine du travail et comme l'auteur de «curieuses recherches sur la physiologie du travail professionnel».
C'est vers la physique médicale, introduite depuis peu dans les facultés de médecine, qu'Imbert se dirige dès le début de sa carrière. Il y est initié par son directeur de thèse, Ferdinand Monoyer (18361912), qui lui confie en 1884 la réalisation d'une nouvelle édition du Traité élémentaire de physique médicale de Wilhelm Wundt, qu'il avait traduit en 1871 (Wund, 1884). Dans les notes qu'il rédige pour cet ouvrage, Imbert manifeste sa connaissance encyclopédique de la physique médicale, depuis l'optique et l'acoustique, jusqu'aux lois du mouvement ou la physiologie des échanges gazeux ou liquides dans le corps humain. On voit là se dessiner le cadre théorique qu'il mobilisa plus tard dans ses recherches sur le travail.

Malgré cet intérêt précoce pour la physiologie, c'est toutefois à la spécialité de sa thèse, l’optique, qu'Imbert se consacre dans la première moitié de sa carrière. Dans les années 1880, il étudie la physiologie de l'œil et diverses anomalies de la vision. Il a le souci de donner à ses recherches une portée pratique, notamment dans l'hygiène scolaire, en diffusant dès le milieu des années 1880 des notions d'optique auprès des instituteurs de la ville de Montpellier(IMBERT, 1887). En 1892, alors qu'il vient d'entrer au conseil municipal de Montpellier(4), il met en place un «service d'inspection oculaire» des écoles communales de la ville, dont l’objectif était de «donner des indications aux parents de chaque écolier, relativement aux professions qui peuvent être nuisibles à la vision de l'enfant ou à celles pour lesquelles celui-ci se trouve frappé à l'avance d'une sorte d'infériorité par suite de quelque cause d'origine oculaire» (idem).

Imbert est aujourd'hui surtout connu des médecins pour ses travaux en radiographie, prolongement naturel de sa formation d'opticien. Il fut en effet l'un des premiers à développer cette technique en France, deux mois après la découverte des rayons $\mathrm{X}$ par l'allemand Roentgen (décembre 1895). Il contribua à la diffusion de ce procédé en France, notamment durant la Première Guerre mondiale, au cours de laquelle il dirigea le service de radiographie de la région militaire de Montpellier (IMBERT, 1915). La radiographie est moins éloignée des questions du travail qu'il y paraît: Imbert y voit un moyen de donner une base scientifique à l'arbitrage des conflits opposant patrons et ouvriers sur l'évaluation de la gravité des dommages corporels entraînés par un accident. L'ayant lui-même expérimentée à des fins d'expertise légale dès 1902 (IMBERT, 1902a), il la conçoit comme une méthode révolutionnaire, permettant «d'adjoindre une certitude aux probabilités de l'examen clinique habituel » (IMBERT, 1910). Henri Bertin-Sans (1862-

(4) Le maire était alors un républicain radical, Ferdinand Castets. Imbert proposa en 1894 un rapport sur les habitations ouvrières et resta au conseil municipal jusqu'en 1898. 
1952) qui fut son assistant pendant plus de vingt ans à la faculté de médecine de Montpellier, affirme que c'est son contact quotidien avec les accidentés du travail, à l'occasion d'examens radiologiques, qui conduisit Imbert à s'intéresser aux problèmes du travail (BERTIN-SANS, 1922).

Outre l'optique, Imbert s'intéressa à une autre branche de la physique médicale: la physiologie du muscle, sur laquelle, il publia, dans le prolongement de sa thèse de physique sur l'élasticité et dans le sillage de Jules Marey (1830-1894) et d'Auguste Chauveau (1827-1917), diverses contributions dans les années 1890. Il donne du mécanisme de la contraction musculaire une explication nouvelle, fondée sur l'inégale répartition des excitations nerveuses dans les parties du muscle, permettant d'accomplir un mouvement avec la moindre dépense d'énergie. À partir de 1903, Imbert se consacre essentiellement aux applications de la physiologie au travail professionnel. Ses recherches en la matière sont reconnues dans le monde médical, puisqu'il obtient en 1911 le prix Chaussier de l'Académie de médecine, en récompense de sa «rigueur scientifique dans l'analyse du travail professionnel» et de «l'action de justice et de pacification qui peut résulter de ces études». En 1922, une chaire de physiologie du travail (la première en France) est créée pour lui à la faculté de médecine de Marseille. Il ne put toutefois développer ce nouveau poste, car il meurt quelques mois plus tard.

Les recherches d'Imbert sur le travail professionnel suscitèrent aussi l'intérêt des milieux politiques. Les socialistes notamment, y voient une légitimation scientifique de leurs revendications. Dans son discours sur la journée de 8 heures du 11 mars 1912 à la chambre, Édouard Vaillant, qui entretenait depuis 1908 une correspondance suivie avec Imbert(5), consacre un long passage aux travaux de ce dernier, qu'il présente comme l'auteur de «recherches très intéressantes», ayant «montré qu'en tenant compte du rapport qui existe entre le travail individuel lui-même et les outils perfectionnés à l'aide desquels il s'effectue, on pouvait obtenir une utilisation meilleure du travail, réduire la durée de ce travail, et par suite, diminuer la fatigue de l'ouvrier » (6). Imbert est également invité par les syndicats ouvriers à participer au cinquième congrès de l'hygiène et de la sécurité des travailleurs et des ateliers (1909), où est évoqué "son dévouement depuis de longues années à la classe ouvrière». Ce congrès fait siens les vœux d'Imbert d'un arbitrage scientifique des conflits du travail et crée à cette fin en 1909 un laboratoire scientifique ouvrier d'études du travail, lieu d'une véritable contre-expertise

(5) Rappelons que Vaillant était lui aussi médecin de formation.

(6) Journal officiel du 12 mars 1912, débats parlementaires, chambre des députés, p. 678 et sq. ouvrière sur les questions d'hygiène et de sécurité. Signalons enfin qu'Imbert s'exprime à diverses reprises dans la presse socialiste et rejoint les positions des syndicats ouvriers sur des questions comme la journée de 8 heures ou l'assimilation des maladies professionnelles aux accidents du travail (IMBERT, 1905).

Il serait toutefois erroné de ranger Imbert parmi les socialistes, car il se montre circonspect face à la perspective d'une solution politique radicale de la question sociale, reposant sur le changement du régime social de propriété. Surtout il veille à ne jamais franchir le seuil de l'engagement politique, car la science n'est pour lui «ni capitaliste, ni socialiste, elle est la vérité même» (IMBERT, Mestre, 1905a). Il précise souvent que ce ne sont pas des convictions politiques qui l'ont conduit sur ses terrains de recherche, mais la combinaison de circonstances fortuites et de la curiosité scientifique. La façon dont il fut amené à travailler sur les coupeuses de sarments de vigne (voir infra) est à cet égard révélatrice: l'une de ses patientes estimant perdre du temps, c'est-à-dire des rétributions salariales, lors de ses consultations à la clinique, il fut amené à l'interroger sur ses méthodes de travail, et se rendit finalement sur place pour observer son travail et celui de ses collègues (IMBERT, 1909). Autant qu'on peut en juger, politiquement, Imbert se situe au carrefour de divers courants réformistes de son temps: le solidarisme de Léon Bourgeois (7), qu'il connaissait personnellement, mais aussi probablement le catholicisme social, comme le laisse penser l'audience privée qu'il obtint du pape Pie X, à l'occasion de sa participation au congrès international des accidents du travail à Rome en 1909 (IMBERT, 1996).

L'engagement social et l'œuvre scientifique d'Imbert sont marqués par un fort ancrage dans la région méridionale, à laquelle il resta très attaché(8). La plupart de ses enquêtes furent réalisées sur des petits métiers de la région de Montpellier: dockers, coupeuses de sarments de vignes, manutentionnaires de la chimie. Son action dépasse toutefois les frontières de sa région et il se place parmi les meilleurs spécialistes d'hygiène et de physiologie du travail au plan international (9). L'hygiénisme est en effet un mouvement qui a d'emblée pris une forme internationale, à travers la tenue de congrès dans

(7) Le thème de la solidarité apparaît notamment dans l'ouvrage le plus personnel d'Imbert, Observations économiques de vies ouvrières (1911). Cet ouvrage fait l'objet d'un compte-rendu par Maurice Halbwachs dans l'Année sociologique (1912), qui ne cache pas sa déception.

(8) Il refusa ainsi de prendre la direction du laboratoire de physiologie du travail du Conservatoire des arts et métiers en 1913, ne souhaitant pas venir s'installer à Paris (archives CNAM, conseil d'administration du 20 octobre 1913).

(9) Ses travaux sont par exemple connus de Max Weber, qui les commente dans Zur Psychophysik der industriellen Arbeit, 1995 (première édition en 1908-1912). 
les capitales européennes dans la seconde moitié du XIX ${ }^{\mathrm{e}}$ siècle dans les divers domaines de l'hygiène sociale (hygiène alimentaire, scolaire, habitation, accidents du travail) (RAsmussen, 2001). Une ambition positiviste d'exhaustivité anime les organisateurs de ces congrès et les volumineux rapports qui en sont issus compilent les idées et données statistiques recueillies dans chaque pays. Imbert cite fréquemment, à l'appui de ses thèses, des statistiques provenant de pays étrangers (10) et ne cesse de rappeler les avancées sociales d'autres pays européens. Ce discours est repris sur la scène politique par Édouard Vaillant, qui déplore que les travaux d'Imbert soient davantage connus à l'étranger qu'en France: "Si la France, qui a sur les autres nations une avance considérable qu'elle doit aux études de ses chercheurs, ne prend pas les mesures que je lui indique, ce n'est plus elle qui aura la direction du progrès qui s'accomplit: c'est l'Allemagne».

Loin de s'enfermer dans le laboratoire, Imbert souhaite faire déboucher ses recherches sur des réglementations : à trois reprises, ses travaux apportèrent une contribution décisive à l'activité réglementaire de l'Office du travail, puis du ministère du Travail dans la période 1900-1914. Ses premières relations avec l'administration du travail se produisirent très certainement au niveau local, à l'occasion de sa rencontre avec Antonin Mestre, inspecteur du travail de l'Hérault depuis 1895. En 1903, l'Office du travail avait demandé à l'ensemble des inspecteurs de mener une enquête sur le transport de charges à l'aide du "cabrouet» (petite brouette à deux roues basses, également appelée diable), afin de savoir si, au vu du décret du 13 mai 1893, les jeunes ouvriers pouvaient être autorisés à utiliser cet instrument. Ce décret fixait en effet à $15 \mathrm{~kg}$ la charge maximale autorisée aux enfants de 14 à 18 ans, mais n'établissait pas de rapport d'équivalence avec les moyens de transport existants. C'était donc aux inspecteurs du travail, souvent ingénieurs de formation(11), d'établir les normes pratiques, non sans susciter parfois des différends avec les employeurs. Ainsi, dans l'Hérault, Mestre avait dû régler en 1901 un conflit à ce propos entre les syndicats de dockers du port de Sète et les compagnies de navigation(12). Mestre et Imbert réalisèrent ensemble une étude qui fut chaleureusement accueillie par le directeur de l'Office du travail, Arthur Fontaine, qui en fit publier les résultats en 1905 dans le Bulletin de l'inspection du travail (Imbert, Mestre, 1905b) et les signala au ministre du Commerce, Fernand

(10) Les courbes décrivant la variation du nombre d'accidents au cours de la journée de travail, qu'Imbert établit avec Mestre en 1904 pour la région de Montpellier (voir infra), s'appuient sur des statistiques établies dès 1897 en Allemagne, voir Emmanuel Rотн, 1907, p. 618.

(11) Sur la formation et le profil des inspecteurs du travail dans cette période, voir notamment Vincent VIET, op. cit., 1994.

(12) AN F22/466.
Dubief (1850-1916). Cette étude inspira l’arrêté du 7 mars 1908 déterminant la charge maximum autorisée au cabrouet pour les enfants de moins de 16 ans. Imbert ne fut toutefois pas pleinement satisfait des conclusions réglementaires de son étude, considérant que d'autres paramètres que la charge, notamment l'état du terrain, déterminaient l'effort productif réel (IMBERT, 1909b).

On voit donc que si Imbert estime nécessaire l'intervention de l'État, il plaide pour des réglementations souples, adaptées au contexte spécifique à chaque travail. En 1910, Arthur Fontaine fait de nouveau appel à lui sur un thème voisin, à l'occasion d'un conflit social opposant des patrons boulangers à des porteuses de pain, traînant des charrettes à bras pesant $150 \mathrm{~kg}$, et chargées de $50 \mathrm{~kg}$ de pain. Imbert propose de «recueillir les appréciations des intéressés et de les soumettre au contrôle expérimental »(13). Parallèlement à son étude, menée in situ sur des sols de nature variée, une recherche sur les propriétés mécaniques des différents modes de charge envisagés était réalisée au CNAM. Les conclusions du rapporteur auprès de la Direction du travail établissent sans conteste l'avantage de l'expérience d'Imbert, qui est «plus directe et plus complète, et dont les conclusions paraissent plus probantes» (idem).

La dernière collaboration entre Imbert et le ministère a lieu en 1913, à l'occasion de la création, par le ministre Henri Chéron, d'une «Commission d'études relatives à la physiologie du travail professionnel, aux conditions de l'existence, aux aptitudes professionnelles et à leur formation dans les familles ouvrières et paysannes » (décret du 17 mai 1913). Celle-ci, forte de vingt-quatre membres, est composée à parité d'hommes politiques ou administrateurs du ministère (Léon Bourgeois, Albert Thomas, Arthur Fontaine, Lucien March...) et de spécialistes du travail (Armand Imbert, Jules Amar, Auguste Chauveau, Henri Le Chatelier, Jean-Paul Langlois...). Imbert est chargé de rendre un rapport sur les études à entreprendre dans le domaine de la physiologie du travail. Il ne s'agit plus, comme précédemment, de mener des enquêtes ponctuelles venant éclairer l'activité réglementaire du ministère, mais de faire une synthèse sur les apports de la physiologie du travail à la gestion des questions sociales, dans l'objectif de créer un véritable service de recherche attaché au ministère.

Le rapport remis par Imbert en octobre 1913 (IMBERT, 1913a) trace un vaste programme de travail: l'alimentation des travailleurs, la fatigue et le repos, l'étude des meilleures techniques professionnelles (par l'observation des ouvriers "virtuoses»). Des thèmes plus originaux, absents des recherches antérieures d'Imbert sont aussi évoqués: l'examen 
précoce des aptitudes psychologiques des enfants, en vue de leur orientation professionnelle et la modification des outillages, afin «d'améliorer ou d'augmenter le rendement», mais aussi « de diminuer la fatigue de l'ouvrier». Les travaux de la Commission de physiologie du travail ne furent finalement pas poursuivis, en raison de la guerre. En revanche, des budgets furent affectés fin 1913 à la création d'un laboratoire de physiologie du travail au CNAM, dont la direction fut confiée à Jules Amar. L’année suivante (juin 1914), il fut également question de créer une chaire de physiologie du travail au Collège de France, à la demande de Vaillant. J.-M. Lahy (1870-1943), dressant quelques années plus tard le bilan des politiques poursuivies par le ministère du Travail déplorait ainsi qu'en dépit «de l'intérêt porté au début du siècle aux problèmes psychologiques du travail» et de la «collaboration que le ministère avait alors établie avec des hommes de science (Imbert, Mestre, Lahy, Frois, etc. ) [...], toutes les propositions qui ont été faites aient été déviées vers l'université» (14), signant par là l'échec d'une véritable «physiologie d'intervention» appliquée au travail.

Les espoirs d'Imbert d'un arbitrage scientifique des conflits entre le capital et le travail, placé sous l'égide du ministère du Travail, furent donc finalement déçus (15). Pourtant, ses conceptions méritent d'être revisitées. En effet, comme nous allons le voir, elles reposent sur une approche épistémologiquement originale du travail, qui conduit Imbert à chercher à déplacer le laboratoire sur les lieux mêmes du travail et à associer directement les ouvriers et les organisations ouvrières à ces recherches. Sa conception de la science du travail débouche alors sur une démarche de «relations professionnelles», fondées sur le dialogue entre employeurs et employés, sous la double égide de la puissance publique et de la science, qui paraît étonnamment en phase avec nos questionnements contemporains.

\section{Énergétisme et économie corporelle}

La façon dont Imbert conçoit l'intervention pacificatrice de la physiologie du travail dans les conflits sociaux est directement liée à la représentation qu’il

(14) Lettre de J.-M. Lahy à Philippe Serre, secrétaire d'État au Travail, 1937 (archives personnelles J.-M. Lahy).

(15) Après la Première Guerre mondiale, c'est vers les patrons qu'Imbert se tourna pour les convaincre de financer des laboratoires susceptibles d'arbitrer scientifiquement les conflits du travail. Une tentative, qui échoua, fut notamment menée en 1921 auprès de la chambre de commerce et d'industrie de Marseille (archives CCI Marseille, cote A 34,12). Ce projet, qui ne put être mené à son terme en raison de la disparition d'Imbert l'année suivante, se prolongea toutefois par la création d'un service d'orientation professionnelle privé au sein de la chambre de commerce de Marseille. a de cette discipline. Si elle s'ancre dans la physique, la physiologie du travail d'Imbert n'est pour autant rien moins que scientiste, car elle met au cœur du raisonnement la sensibilité du sujet travaillant. Il ne saurait donc être question de construire une science du travail sans organiser une collaboration étroite entre le physiologiste et le travailleur, qui n'est pas un simple objet passif de l'enquête, comme il en est, par exemple, dans les études de Jules Amar (18791935)(16), mais bien un agent actif, car «nous sommes nous-mêmes le mécanicien du moteur que nous constituons » (IMBERT, 1902b, p. 9).

Les recherches d'Imbert se situent dans le prolongement de l'analyse mécanique du travail humain, fondée à la fin du XVIII ${ }^{\mathrm{e}}$ siècle par Charles-Augustin Coulomb (1736-1806), sous le «haut patronage» duquel il inscrit lui-même ses travaux (IMBERT, 1911b). Ses sources immédiates sont les recherches complémentaires de deux auteurs étroitement liés: Jules Marey et son analyse chronophotographique des mouvements animaux d'une part, Auguste Chauveau et son étude thermodynamique de la fonction musculaire de l'autre. Il maîtrise aussi, comme tous ses contemporains, les techniques d'enregistrement graphique mises au point par la physiologie allemande du XIX ${ }^{\mathrm{e}}$ siècle, soit la myographie de Karl Ludwig (1816-1895), complétée par l'ergographie de son disciple, l’Italien Angelo Mosso (1846-1910)(17). Mais on ne trouvera nulle trace chez Imbert du physicalisme qui marque l'œuvre de Gustave Adolphe Hirn (1815-1890), lequel, dans les années 1850, ne pouvait concevoir de dépense énergétique sans production de travail extérieur apparent(18), mais aussi celle de son contemporain Jules Amar, qui résume la question à la mesure du «rendement de la machine humaine». On ne trouvera pas non plus ce fétichisme de l'inscription graphique, qui caractérise les abondants et décevants travaux des spécialistes de l'ergographie, tels ceux de Josepha Joteyko (1866-1928), physiologiste belge d'origine polonais (voir à ce sujet VATiN, op. cit., 1999).

(16) Jules Amar soutient en 1909 une thèse sur Le rendement de la machine humaine, Paris, Baillère. Cette étude, menée dans le sillage de la bio-énergétique de Chauveau (voir infra) et financée par une mission du tout jeune ministère du Travail, s’appuyait sur des expériences réalisées sur des prisonniers à la prison de Biskra en Algérie. On est là très loin de l'esprit des enquêtes prônées par Imbert: sur les lieux de travail et avec la collaboration active des ouvriers. Sur les sciences du travail au carrefour des XIX ${ }^{\mathrm{e}}$ et XX ${ }^{\mathrm{e}}$ siècle, voir Georges RibeiLl, 1980, op. cit. pp. 3-36; Anson RabinBaCh, 1990; François Vatin, 1999.

(17) La «myographie» consiste à mesurer le travail mécanique accompli lors d'une action musculaire provoqué par un stimulus, électrique ou chimique. L' «ergographie», mesure de même un travail mécanique produit, mais sur la base d'une action volontaire (stimulus nerveux).

(18) Hirn ne parvient pas à concevoir le «travail intérieur» du muscle, qui sera mis en évidence par J.-B. Chauveau (1888). 
Pour Imbert, la mécanique et la thermodynamique ne sont que des instruments pour dégager des principes d'économie corporelle, dont il a bien conscience qu'une large partie lui échappe encore. C'est pourquoi la recherche doit d'abord s'appuyer sur l'observation du travailleur in situ, dans les conditions ordinaires de son activité. Le travailleur dispose en effet d'une conscience intuitive de son économie corporelle, il est un "physiologiste inconscient, qui dépense sa propre énergie » (IMBERT, 1909d). Cette économie corporelle n'est pas spontanée, elle est produite par l'apprentissage(19) et la marque de la «supériorité professionnelle». Les meilleurs travailleurs sont donc aussi ceux qui savent se fatiguer le moins. Le travail professionnel, convenablement observé, constitue donc une donnée pour le physiologiste. Charge à ce dernier de dégager les modèles théoriques aptes à rendre compte de cette économie corporelle. Dans certains cas, la solution n'exige qu'une analyse cinématique du mouvement, comme dans l'exemple qu'il emprunte à Samuel Haughton sur le choix par les pêcheuses d'un chemin énergétiquement optimal pour remonter de la mer (IMBERT, op. cit., 1902b, p. 17 et sq)(20). Ces femmes devaient choisir entre un chemin direct sur un sol sablonneux (et dont le parcours était donc énergétiquement coûteux), un chemin allongé, qui minimisait la fraction sablonneuse du parcours, ou une combinaison variable entre ces deux itinéraires. Le chemin mixte adopté par les pêcheuses obéit à une loi géométrique simple, familière à l’ophtalmologiste qu'est Imbert, puisqu'elle est formellement analogue à la "loi élémentaire de réfraction», qui correspond à la minimisation du temps mis par un rayon lumineux pour aller d'un point à un autre (IMBERT, idem, p. 19).

Cet exemple est simple, parce qu'il ne nécessite pas une analyse thermodynamique de la fonction musculaire, mais aussi parce qu'il correspond à une activité globale de l'organisme que constitue la marche. On peut alors raisonner en termes de dépense énergétique globale, comme le fera par exemple Jules Amar. Mais Imbert est bien conscient que tel n'est pas le cas général du travail professionnel et que, de ce fait, les travaux sont énergétiquement incomparables les uns aux autres. Il fournit une illustration suggestive de cette idée en comparant les travaux mécaniques extérieurs respectivement fournis par un facteur rural faisant à pied deux tournées de trois heures par jour (259200

(19) La place nous manque ici pour développer ce point extrêmement important dans la physiologie du travail d'Imbert. Le mécanisme d'apprentissage repose selon lui sur la sensibilité musculaire dont la sensation de fatigue constitue le signal significatif pour l'organisme: «Que les lois mécaniques de l'acte à accomplir aient été établies, ou qu'elles nous soient au contraire totalement inconnues, c'est toujours par la fatigue que nous nous déterminons» (op. cit., 1902, p. 27).

(20) Imbert s'appuie sur Samuel Haughton, Principles of animal mechanics, Londres, 1873. kilogrammes mètres), un ouvrier de chais travaillant à la pompe (212200 kilogrammes mètres), un docker charbonnier du port de Sète travaillant neuf à dix heures (75000 kilogrammes mètres), pour conclure: «Il est hors de contestation [...] que ces diverses professions sont en réalité d'autant moins fatigantes que le travail y est évaluable par un plus grand nombre de kilogrammètres » (IMBERT, 1907, pp. 245-259: 251)(21)! La raison de ce paradoxe est double: d'une part, la dépense énergétique est à rapporter à la nature de la musculature mise en jeu et, par exemple «ce sont les puissants muscles des membres inférieurs qui travaillent pendant la marche, tandis que le travail des ouvriers charbonniers est surtout effectué par les muscles moins volumineux et plus grêles des membres supérieurs » (idem, p. 250). Mais, par ailleurs, la dépense énergétique globale du muscle n'est pas directement corrélée avec la fatigue produite, qui dépend de la distribution de cette dépense dans le temps: un effort continu moyen (celui de l'ouvrier des chais travaillant avec un levier) étant moins fatigant qu'un effort brusque élevé (celui du docker qui doit soulever des poids).

Mais ces considérations n'épuisent pas le problème. Inspiré par Chauveau, Imbert est sensible à la question du rendement énergétique du muscle lui-même (la plus ou moins grande déperdition de travail en chaleur), qui est variable selon les situations de travail. Enfin, pour être complet, il faudrait selon lui tenir compte de la dépense d'énergie neuromusculaire aperçue par Chauveau. Pour être énergétiquement très réduite, celle-ci ne doit pas pour autant être négligée, puisque l'important à son sens n'est pas tant la valeur absolue de l'énergie dépensée par un organe que le rapport entre la valeur moyenne dépensée par cet organe et les capacités maximales qu'il peut développer (ImBert, op. cit., 1902 b). Mais Imbert a bien conscience qu'une telle mesure est encore hors de sa portée(22). En conséquence, si, tout travail, comme toute activité vitale, a bien pour lui une dimension énergétique, la mesure énergétique du travail, telle qu'on peut pratiquement la mettre en œuvre, n'a pas pour autant une portée universelle. Il serait par exemple «absurde [...] de croire qu'une telle évaluation peut conduire à une appréciation suffisamment exacte du labeur d'un mécanicien conduisant une locomotive, ou d'un ouvrier de l'industrie surveillant des métiers à tissage» (IMBERT, 1906, pp. 521-524: 522).

(21) Imbert emploie le terme «kilogrammètres» pour désigner une unité de «travail» (produit d'une force par le déplacement du point d'application de cette force) correspondant à des kilogrammes multipliés par des mètres.

(22) Cette question fera toutefois l'objet de sa dernière note scientifique: «Mode de fonctionnement économique du cerveau », Année psychologique, 1922, pp. 221-223. 
Autrement dit, pour Imbert, il n'est pas possible de « descendre » d'une théorie générale de la dépense énergétique à la sensation de fatigue des travailleurs ; il faut, au contraire, «remonter » de la connaissance de celle-ci à l'analyse des mécanismes musculaires et neuromusculaires, pour mieux comprendre la si compliquée organisation du moteur humain. «La considération de la fatigue, malgré le peu de précision absolue de ce procédé de mesure, est beaucoup plus utile que celle du travail, au sens purement mécanique du mot» affirme-t-il déjà en 1902, alors qu'il n'a pas encore réalisé d'études sur le terrain (IMBERT, op. cit., 1902b, p. 15). Six ans plus tard, il pourra préciser les choses, en invitant à aller étudier in situ la fatigue ouvrière «en pénétrant dans l'atelier et dans l'usine pour y observer et y étudier le travail pendant que l'ouvrier l'effectue. La constatation des faits est d'autant plus intéressante et utile qu'on la fait servir à la découverte des causes, et du fait fatigue, on doit remonter à la cause travail». (IMBERT, 1908b, pp. 232-248: 247).

Si la comparaison mécanique des travaux n'est pas possible en toute généralité, elle l'est quand les données du problème sont assez stables. Imbert en donnedeuxexemples.Lepremierconcernelecoupage des sarments de vigne. Les femmes employées à cet effet étaient payées aux pièces, selon deux tarifs différents dépendant du diamètre des boutures: « 0 fr 50 par 1000 boutures, dites petites, d'un diamètre égal ou inférieur à 6 millimètres, et 0 fr 60 par 1000 boutures, dites grosses, d'un diamètre supérieur à 6 millimètres » (IMBERT, op. cit., 1907, p. 50). Or, elles se plaignaient de l'insuffisance de ce second tarif, eu égard à la plus grande fatigue du travail. Après analyse mécanique du travail, Imbert conclut que «les réclamations des ouvrières étaient incontestablement justifiées », puisque: «Au prix de 0 fr 50 les 1000 petites boutures, chaque centime de salaire correspond à une somme d'efforts partiels égale à $110 \mathrm{~kg}$ ", alors que «au prix de $0 \mathrm{fr} 60$ les 1000 grosses boutures, au contraire, l'ouvrière doit réaliser une somme d'efforts partiels égal à $266 \mathrm{~kg}$ par centime de salaire»(23). Le second exemple concerne le choix des techniques. Imbert compare la fatigue induite par l'usage de la brouette ou celui du cabrouet (IMBERT, Mestre, op. cit., 1909) à partir de variables physiologiques (nombre d'inspirations et de pulsations par minute), pour conclure sans hésitation que l'emploi de la brouette est «plus fatigant que celui du cabrouet, auquel on devra donner par suite la préférence, à moins que quelque

(23) Idem, p. 53. Comme il le souligne ce résultat ne permet pas pour autant de fixer une norme juste de salaire, puisqu'on pourrait tout autant baisser le tarif pour les petites boutures qu'augmenter celui pour les grosses. Comme on le verra plus loin, sa théorie du salaire obéit à d'autres logiques. En l'occurrence il signale que la direction de l'entreprise évita la grève en fixant un tarif de la coupe à $0,65 \mathrm{fr}$ les 1000 boutures, quelles qu'en soient les tailles. raison particulière ne puisse être invoquée contre ce choix» (IMBERT, op. cit., 1907, p. 61).

La finesse de la combinaison par Imbert des points de vue énergétiste et socio-économique se fait jour dans sa théorie du salaire. Il a bien conscience qu'ouvriers et patrons n'ont pas la même représentation du salaire: les patrons le conçoivent comme la contrepartie du travail fourni alors que les salariés le conçoivent comme la contrepartie de la fatigue subie. Or, selon Imbert, ces deux variables varient en sens inverse en fonction du temps, puisque la productivité marginale du travail tend à baisser dans le cours de la journée, alors qu'à l'inverse la fatigue marginale augmente: «En conséquence, le salaire horaire devrait diminuer d'heure en heure au point de vue patronal et augmenter, au contraire, au point de vue ouvrier» (IMBERT, op. cit, 1911b, p. 479).

Or Imbert montre, à partir d'une analyse énergétique, que le point de vue patronal ne tient pas et rejoint curieusement par ce biais la théorie marxiste du salaire. Prenant le cas du facteur rural se déplaçant à bicyclette et payé 4 francs par jour, il montre que la dépense énergétique liée au travail mécanique fourni correspond à la consommation d'environ 945 calories alimentaires, alors que la ration de repos est d'environ 2300 calories: «Donc, à ne considérer le salaire que comme l'équivalent physiologique du travail fourni, c'est seulement 0 fr 26 que l'on devrait donner au facteur considéré, au lieu des 4 francs qu'il touche en réalité journellement» (idem, p. 480). Autrement dit, «il y a donc, dans le salaire, autre chose que la rémunération, brute en quelque sorte, du travail professionnel envisagé au point de vue physiologique. C'est qu'en effet pour pouvoir travailler, il faut d'abord être vivant, et nul employeur ne peut dès lors se refuser à concéder que le salaire ne doit pas seulement correspondre aux 945 calories exigées par le travail professionnel, mais encore aux 2300 calories qui sont la ration journalière de l'ouvrier au repos » (idem). Poursuivant l'analyse, il montre, à la manière de Marx, que le salaire doit couvrir l'ensemble des éléments de «reproduction de la force de travail»: le vêtement, le logement, l'entretien de la famille...

La physiologie du travail d'Imbert ne se referme donc pas sur elle-même comme une science positive qui déboucherait mécaniquement sur des solutions sociales. Le rapprochement qu'il préconise entre la physiologie et les sciences économiques et sociales n'est pas à sens unique. Sans doute, comme il l'affirme en 1907, alors que le ministère du Travail vient d'être créé, la société doit prendre en considération les résultats de la physiologie pour procéder à ce travail d'élaboration normatif: «Il me paraît [...] juste, rationnel et nécessaire [...] de tenir compte de la somme des renseignements pouvant être fournis par les médecins et les physiologistes pour l'élaboration des règlements et des lois qui visent le travail professionnel » (IMBERT, op. cit., 1907, p. 258). Mais, 
réciproquement, la physiologie du travail ne saurait être refermée dans le cadre confiné du laboratoire: «Dans ce ministère du Travail humain, une place doit être réservée à l'étude de l'homme au travail. Et cette étude doit être faite dans des conditions plus directement pratiques que celles qui président à des recherches de laboratoire. C'est sur l'ouvrier luimême que doivent porter les observations, c'est sur le chantier et à l'usine que les recherches doivent être poursuivies » (idem).

Seule une telle recherche peut déboucher pour Imbert sur des connaissances opérationnelles. Mais, comme on va le voir, un tel mode de recherche a aussi par lui-même une valeur pacificatrice dans le conflit capital-travail. La physiologie du travail d'Armand Imbert débouche ainsi sur un esprit de recherche-action, qui n'oppose pas, mais combine au contraire étroitement positivisme scientifique et organisation du dialogue social.

\section{Arbitrage scientifique et dialogue social}

Toute l'œuvre d'Imbert en matière de médecine du travail est tournée vers la recherche de mécanismes d'arbitrage entre ouvriers et patrons fondés sur une science physiologique du travail. Mais sa conception de l'intervention de la science débouche sur une logique de "relations professionnelles", dans l'esprit du cadre juridique nouveau dans lequel se déploient les relations de travail en France au moment où est créé le ministère du Travail (voir sur ce contexte, DIDRY, 2002).

C'est à partir de la question des accidents du travail qu'Imbert a été amené à développer cette problématique. La loi du 9 avril 1898 avait imposé aux employeurs qui y étaient assujettis une cotisation obligatoire à des compagnies d'assurance pour couvrir les frais médicaux et les «demi-salaires» à verser aux ouvriers en cas d'incapacité. De là découlait un abondant contentieux tripartite entre les employeurs, les assureurs et les ouvriers: «Les ouvriers en effet récriminent contre la rigueur des compagnies d'assurance à leur égard; les compagnies se plaignent d'être exploitées par les ouvriers, et les patrons déplorent l'accroissement des charges qui leur incombent, par suite du relèvement des primes d'assurance » (IMBERT, 1904). Les médecins étaient mobilisés comme experts pour évaluer les préjudices, donnant éventuellement droit à une rente, et les périodes d'incapacité donnant droit au demi-salaire. Ils se trouvaient donc au cœur du conflit(24).

Imbert intervient dans ce débat sur plusieurs registres. D'une part, il bat en brèche l'idée que

(24) Voir F. Pons (1912), conseil judiciaire de la Bourse du travail (CGT), qui recommande notamment aux accidentés: «Refusez le médecin de la compagnie ou du patron» (p. 78). les ouvriers retarderaient par paresse leur retour au travail en soulignant que rares sont ceux qui peuvent se contenter de bon cœur d'un demi-salaire(25). Son analyse repose sur une étude statistique de la fréquence des accidents du travail selon les heures de la journée et les jours de la semaine pour défendre l'idée que la fatigue, croissante avec la durée du travail, serait la cause majeure de ceux-ci. Il s’appuie sur des enquêtes menées dans l'Hérault avec Antonin Mestre, qu'il complète par des données réunies par M. Le Roy, inspecteur divisionnaire à Toulouse, ainsi qu'avec des statistiques officielles belges.

Ses calculs le conduisent à conclure que « toutes les statistiques démontrent [...] que le nombre des accidents augmente progressivement d'heure en heure, au cours des demi-journées quotidiennes » (IMBERT, op. cit., 1908b, p. 239) et à recommander des pauses intercalaires au sein de chaque demi-journée de travail (IMBert, 1904, p. 716; ImBert, Mestre, 1905, pp. 520-526: 521). Mais ces études le conduisent également à préconiser un cadre paritaire d'arbitrage avec l'instauration "d'ouvriers inspecteurs », désignés par les syndicats ouvriers, «dont toutes les journées prises par la surveillance à exercer seraient rétribuées par les compagnies» (IMBERT, 1908a, pp. 97-122: 120). À plus long terme, il envisage que les syndicats ouvriers soient intéressés au bénéfice des compagnies d'assurance, de même que les patrons bénéficieraient d'un système de primes modulables (bonus-malus) suivant les résultats. Il s'agit pour lui, au-delà du traitement comptable de la question, de responsabiliser chacune des parties pour une diminution de la dangerosité du travail. Il souligne d'ailleurs que les compagnies d'assurance et les ouvriers ont en la matière des intérêts convergents: « Je crois que c'est une erreur profonde de regarder comme adversaires naturels les ouvriers d'une part, les compagnies d'assurance de l'autre. Celles-ci se sont-elles jamais demandées dans quelle proportion leurs dividendes étaient augmentés par la victoire des ouvriers à la suite d'une grève? Qu'il s'agisse d'une augmentation de salaire qui équivaudra à une augmentation du bien-être des travailleurs et, par suite, à une augmentation de leur résistance à la fatigue, ou d'une diminution des heures de travail qui se traduira par une moindre fatigue des ouvriers, les compagnies d'assurances sont, dans une certaine mesure, les bénéficiaires des revendications au sujet desquelles le prolétariat a obtenu gain de cause» (IMBERT, op. cit., 1904, p. 71).

Un raisonnement semblable est à l'œuvre dans l'analyse faite par Imbert des maladies professionnelles. À la demande du Moniteur des syndicats ouvriers, il s'exprime en 1905 sur ce sujet, qui est alors au cœur d'un débat entre l'administration du

(25) A. Imbert souligne en revanche que les ouvriers peuvent, notamment quand ils sont âgés, avoir pour stratégie l'obtention d'une rente (op. cit., 1904, p. 713). 
travail et les syndicats ouvriers (sur ce point, voir VIET, RufFAT, op. cit., 1999, pp. 25-31). Ces derniers réclament que les maladies contractées dans le cadre professionnel donnent lieu à indemnités, au même titre que les accidents du travail. L'administration du travail hésitait à légiférer sur cette question en raison de ses difficultés pratiques d'application. En effet, à l'exception des maladies par intoxication(26), il était difficile d'imputer la responsabilité d'une maladie à un employeur précis (les ouvriers se déplaçant fréquemment d'un établissement à l'autre) et de démêler les causes professionnelles et extra-professionnelles de l'apparition d'une maladie. Imbert raisonne en deux temps: il suggère d'abord de recueillir des données statistiques pour identifier les industries qui se caractérisent par une surreprésentation de certaines maladies chez leurs ouvriers; il encourage ensuite les employeurs de chaque industrie à contracter une assurance collective dont bénéficieraient tous les ouvriers de l'industrie en question. La date d'apparition de la maladie cesserait alors d'être un objet de contentieux entre ouvriers et employeurs.

L'analyse d'Imbert se caractérise par une grande sensibilité sociologique, non seulement dans son usage discriminant des statistiques, qui n'est pas sans rappeler celui de Durkheim, mais aussi par son analyse du droit. Il considère en effet que la législation doit modeler les pratiques des acteurs sociaux, et non l'inverse: examinant les objections habituellement faites à l'encontre d'une loi sur les maladies professionnelles il écrit: "Quelle est la loi qui ne présente pas, à un degré plus ou moins accusé, un tel caractère d'imperfection? [...] des objections analogues peuvent, en particulier, être faites à la loi du 9 avril 1898 sans, pour cela, qu'elle cesse de constituer un important progrès social» (IMBERT, 1905).

Imbert ne conçoit donc pas l'intervention de l'expertise scientifique selon le modèle philanthropique classique où l'ouvrier est considéré comme un être psychologiquement mineur, ayant une fausse conscience de ses intérêts, et que le bienfaiteur éclairé doit d'abord rééduquer. Selon cette représentation, qui débouche, chez les médecins hygiénistes comme chez les ingénieurs leplaysiens, sur une prophylaxie sociale dans l'espace domestique ouvrier, il faudrait protéger l'ouvrier, d'abord contre lui-même et ses mauvais penchants (alcoolisme, sexualité). A contrario, Imbert souligne que «l'indifférence presque hostile» avec laquelle cette "médecine sociale» a été reçue dans les milieux syndicalistes vient de ce que ceux-ci récusent le postulat selon lequel les causes principales de la

(26) Principalement le phosphorisme, qui touchait les employés des fabriques d'allumettes, et le saturnisme, qui touchait les peintres en bâtiment ou certains ouvriers de l'industrie chimique. La reconnaissance de ces maladies comme maladies professionnelles ne sera acquise que par la loi du 3 juillet 1913 . morbidité ouvrière seraient à chercher dans les conditions de vie hors travail (logement, alcoolisme), car elles résident selon eux dans le travail lui-même ("surmenage») et dans la faiblesse des salaires (IMBERT, 1907, op. cit., pp. 245-246). À l'écoute du discours ouvrier, Imbert entend donc faire porter le regard prophylactique sur l'espace du travail lui-même. C'est dans cet esprit d'une philanthropie renouvelée par le républicanisme social qu'il préside la section de l'Hérault de l'Alliance d'hygiène sociale, dont le président national est alors Léon Bourgeois: "Tout en poursuivant son ouvre féconde en ce qui concerne l'hygiène physique, l'Alliance d'hygiène sociale pourrait utilement étendre son action impulsive vers l'étude des dépenses d'énergie auxquelles l'ouvrier doit satisfaire » (idem, p. 84) (27).

La recherche d'un arbitrage scientifique n'est donc pas chez Imbert opposée à la mise en œuvre d'un «dialogue social». Les deux sont au contraire à mettre en synergie. Ainsi, en opposition à la conception dominante de l'époque, qui voit dans l'hygiène industrielle un instrument d'intervention entre les mains de l'État protecteur, chargé de définir des normes de travail exogènes aux régulations ouvrières, Imbert fonde la légitimité de ses méthodes sur le dialogue social entre représentants des syndicats ouvriers et du patronat. En cela il apparaît comme un précurseur de l'ergonomie des années 1970, telle qu'elle s'exprime au moment de la création de l'Association nationale pour l'amélioration des conditions de travail (ANACT), créée en 1973, qui cherche elle aussi à construire un consensus entre partenaires sociaux autour du thème des conditions de travail.

Ce caractère novateur s'exprime particulièrement bien dans la place qu'Imbert entend donner aux partenaires sociaux dans les recherches scientifiques sur le travail. Les ouvriers, premiers intéressés par les résultats des études physiologiques sur le travail, doivent être impérativement associés aux discussions. Ainsi, faisant un compte rendu de la session consacrée au "surmenage par suite du travail professionnel» du IV e congrès international d'hygiène et de démographie tenu à Berlin en septembre 1907, Imbert regrette l'absence d'un rapporteur ouvrier: "Peut-être eût-il été désirable, en toute impartialité, qu'aux quatre rapporteurs seuls désignés [trois chercheurs en médecine dont lui-même et l'ingénieur en chef du service des eaux de Berlin, dont il critique la contribution qui reprend les lieux communs de la pensée patronale], on eût

(27) À l'occasion de cet exposé tenu au congrès de l'Alliance d'hygiène sociale à Roubaix en septembre 1911, Léon Bourgeois a rendu hommage au projet d'Imbert: «Il y a je dirai presque le germe d'une politique sociale portant directement sur une science dont nous ne pouvons pas méconnaître l'importance et dont les conséquences sont peut-être extrêmement étendues et presque infinies » (cité par Marcel Turbiaux, 2003). 
adjoint un cinquième qui eût été le représentant des ouvriers, puisque c'est d'eux surtout qu'il allait être question" (IMBERT, op. cit. 1908b, p. 232). Deux ans plus tard, il revient à la charge sur ce thème, en rappelant par exemple qu' "en 1903, à Bruxelles, la discussion de l'ankylostomasie, par exemple, question cependant essentiellement médicale, devint particulièrement intéressante lorsqu'elle se limita entre le directeur des mines, M. Harzé, et un ouvrier mineur, M. Cavrot» (IMBERT, 1909a, pp. 574-578: 575). Il rappelle à cette occasion qu'il avait obtenu en 1905 «des municipalités de Montpellier et de Sète, le vote des minimes sommes nécessaires pour l'envoi [au I ${ }^{\mathrm{er}}$ congrès international médical des accidents du travail à Liège] de deux délégués ouvriers, dont le choix fut d'autre part laissé à la libre disposition des bourses du travail de ces villes » (28). Mais les ouvriers ne sont pas les seuls invités à venir exprimer leur point de vue aux côtés des savants. Les patrons doivent aussi y participer, pour autant qu'ils acceptent de venir sans préjugés. Ainsi, en réponse au rédacteur de la Revue industrielle, qui avait critiqué les résultats de ses recherches sur les accidents du travail(29), Imbert répond: "Le rédacteur de la Revue industrielle nous a paru envisager a priori avec crainte les recherches du genre de celles que nous avons entreprises, être prédisposé à en combattre les conclusions, penser peut-être qu'elles ont été commencées avec quelque idée préconçue, et il serait très regrettable que cette opinion fût générale dans le milieu patronal industriel [...] il est à souhaiter que les chefs de chantier et d'usines viennent prendre part aux discussions [des congrès scientifiques] et y verser la somme de renseignements que leur aura fournie leur observation journalière» (ImBERT, Mestre, 1904, pp. 449450: 450).

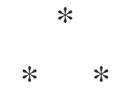

Ainsi, Imbert se fait simultanément l'apôtre de l'arbitrage par la science et du dialogue social : « La solution d'une question complexe ne saurait être définitive, s'il n'est pas tenu un légitime compte de considérations multiples et plus ou moins rigoureusement contradictoires; cette œuvre difficile doit être laborieusement préparée par les individus

(28) Idem, p. 576. Cette initiative fait l'objet d'un article enthousiaste des deux ouvriers, Niel et Jeannot dans la presse de l'Union départementale CGT de l'Hérault Le travailleur syndiqué, en juillet 1905. Voir à ce sujet Georges RibeiLl, 1986, pp. 50-58.

(29) Le rédacteur de la revue reprochait notamment à Imbert et Mestre de fonder leur théorie du surmenage sur «les données de la statistique, bonne fille s'il en fut, offrant ses chiffres à qui veut s'en servir, mais ne pouvant, comme la plus belle fille du monde, ne donner que ce qu'elle a» (Revue industrielle, 8 octobre $1904, n^{\circ} 41$, p. 408). eux-mêmes, isolément d'abord, contradictoirement ensuite, avant que n'interviennent les débats d'où sortira la sanction sociale» (IMBERT, 1909a, p. 577).

Mais ce point de vue est directement soutenu par sa conception même de la science du travail dont il n'envisage pas l'élaboration sans l'observation directe du travail et l'écoute du travailleur. Par ellemême, cette démarche de recherche en collaboration avec les travailleurs a, à son sens, une dimension pacificatrice: "Aller ainsi spontanément vers le travailleur pour s'enquérir de son travail, c'est créer tout d'abord comme une atmosphère de confiance, c'est écarter probablement, lors des discussions à venir, toute idée préalable d'intransigeance, de violence ou de haine, c’est préparer, le cas échéant, l'acceptation d'un arbitrage scientifique désintéressé» (IMBERT, 1912, p. 84).

Un siècle plus tard, la parole d'Imbert nous semble étrangement proche. On n'y trouve nulle trace en tout cas du scientisme souvent reproché à la science du travail professionnel du début du $\mathrm{XX}^{\mathrm{e}}$ siècle (voir par exemple RabinBach, op. cit., 2004). Cette approche originale du travail fut longtemps occultée, pour des raisons qui tiennent en partie au développement dans l'entre-deux-guerres d'une discipline alternative, la psychotechnique, qui connut un important développement autour du mouvement de l'«orientation professionnelle» et parvint à s'imposer pour la sélection et l'orientation des travailleurs dans quelques grandes administrations ou entreprises publiques (Huteau, LaUtrey, 1979, pp. 3-43). Si les conceptions de certains de ses initiateurs, comme Jean-Maurice Lahy (18701943) et Henri Piéron (1881-1964), s’inscrivent dans la continuité directe de la pensée d’Imbert, notamment par l'attention portée à l'observation directe du travail, leur pensée fut souvent trahie par leurs continuateurs dans le monde de l'entreprise (MOUTET, 2004, pp. 83-101) qui utilisèrent ces méthodes de façon mécanique en les intégrant aux pratiques gestionnaires des services du personnel (OMNEs, 2002). La psychotechnique, telle qu'elle se développa en entreprise ou dans les services de Formation professionnelle accélérée (FPA) de l'ANIFRMO(30) entre la fin des années 1930 et le début des années 1970, apparaissait exclusivement centrée sur l'idée de "prédisposition individuelle» et donc nettement en retrait par rapport à la science du travail d'Imbert, qui envisageait l'interaction entre le travailleur et son environnement de travail

(30) Association nationale interprofessionnelle pour la formation rationnelle de la main-d'œuvre, créée en 1947 dans l'orbite du ministère du Travail, pour assurer la formation professionnelle accélérée de la main-d'œuvre, notamment dans le bâtiment et l'industrie. 
de façon plus complexe(31). Une autre raison de l'occultation de la pensée d'Imbert tient certainement à l'introduction du taylorisme en France, qui par la simplicité des formes d'analyse du travail qu'il proposait se révéla un sérieux concurrent aux approches physiologiques du travail dominantes dans la période antérieure(32). Celles-ci étaient en effet soucieuses de fonder les normes de travail sur une analyse scientifique rigoureuse, bien loin du «bon sens » patronal que Taylor se proposait simplement de rationaliser. Dans son dernier ouvrage, paru peu après la guerre, Imbert lui-même présente une analyse très nuancée du «système Taylor » (IMBERT, 1920), nettement moins critique en tout cas que l'ouvrage de LAHY (1916)(33) paru quelques années plut tôt(34).

La science physiologique du travail réapparut toutefois après-guerre sous l'expression d' «ergonomie», terme emprunté à l'anglais Ergonomics, qui fit son entrée en France vers le milieu des années 1960 (35). Cette discipline, dédiée à l'étude scientifique des situations de travail et des relations entre l'homme et la machine chercha elle aussi à se constituer autour de l'observation directe du travail et à développer une "physiologie d'intervention» assez similaire dans sa démarche à celle voulue par Imbert(36). Comme chez lui, il ne s'agit pas en effet de construire un corpus de savoirs théoriques séparés de leurs applications pratiques sur les lieux de travail, mais de faire au contraire appel à la collaboration de différentes disciplines (sciences de l’ingénieur, médecine, physiologie, psychologie...) pour transformer l'environnement de travail.

Cette priorité donnée à la recherche de terrain sur les modèles élaborés en laboratoire, cette attention portée à la demande sociale et à ses différentes composantes, dessinent un fil rouge qui parcourt l'école ergonomique française depuis les premiers travaux d'Imbert jusqu'aux courants les plus récents de l'ergonomie contemporaine. Chez Imbert comme chez ses successeurs, le travail n'est pas un objet statique, qui se laisserait enfermer dans des modèles

(31) À cet égard, les sources épistémologiques d’Imbert semblent se situer davantage dans le lamarckisme, attentif aux interactions entre l'organisme et l'environnement, que dans le darwinisme.

(32) Sur le débat entre les physiologistes du travail et le taylorisme voir F. Vatin, op. cit., 1999.

(33) Cet ouvrage reprenait en fait une série d'articles parus en 1913, soit sept ans avant l'ouvrage d'Imbert.

(34) Rappelons qu'entre la date de parution des deux ouvrages, le taylorisme apporta une contribution décisive à l'effort de guerre, notamment dans les industries d'armement à l'initiative du ministre de l'Armement Albert Thomas.

(35) L’origine de ce terme était en réalité plus ancienne et remontait au milieu du XIX ${ }^{\mathrm{e}}$ siècle. Voir sur ce point VATIN, 2006, pp. 52-62.

(36) En cela, Imbert, peut être considéré comme un précurseur de la «recherche action» dont se réclament de nombreux ergonomes, dans le sillage des travaux d'Alain Wisner ou de Maurice de Montmollin. théoriques a priori, mais un processus dynamique, en mouvement permanent, qui appelle une coopération sans cesse renouvelée entre les experts et les travailleurs dans les lieux de travail eux-mêmes. L'œuvre d'Alain Wisner en porte particulièrement la marque, qui écrivait récemment que «l'idée majeure de l'ergonomie de langue française est que la question est posée par le terrain»(37). Pourtant, si les hommages rendus par l'ergonomie contemporaine à des psychologues du travail tels que Suzanne Pacaud ou J.-M. Lahy sont fréquents, Imbert est étrangement passé sous silence, alors même qu'il a profondément inspiré ces deux auteurs et leurs héritiers (38). Nous espérons avoir montré la fécondité et l'actualité toujours vivante de sa pensée, dont les prolongements se situent tout aussi bien du côté de l'ergonomie que de celui de la psychopathologie du travail ou de la «clinique de l'activité », disciplines toutes deux soucieuses de saisir la singularité des situations de travail, de privilégier la rencontre avec les travailleurs dans leurs situations concrètes de travail et non d'établir des normes extérieures au processus de travail lui-même(39).

(37) Sur la résonance de la pensée d’Imbert dans l'ergonomie de Wisner, voir notamment Duraffourg, Vuillon (sous la direction de), 2004.

(38) Voir notamment l'hommage rendu par Lahy à Imbert, à l'occasion de la publication d'un article posthume de ce dernier dans le premier numéro de la Revue de la science du travail qu'il venait de créer avec le Belge P. Sollier et le Luxembourgeois J.-P. Arend (A. Imbert, 1929, nº 1, pp. 197-216).

(39) Voir à ce sujet Christophe DeJours, 2006, pp. 207-218. 


\section{Bibliographie}

Amar J. (1909), Le rendement de la machine humaine, thèse, Paris, Baillère.

Bertin-Sans (1922), «Nécrologie d'Armand Imbert», Marseille médical, 15 juillet, pp. 693-699.

Bourdelais P. (éd.) (2001), Les hygiénistes. Enjeux, modèles et pratiques (XVIII ${ }^{e}-X I X^{e}$ siècles), Paris, Belin.

Chauveau J.-B. (1888), «Du travail physiologique et de son équivalence», Revue scientifique, n 5 pp. 129-139.

Dejours C. (2006), «Science du travail et politique. À partir de l'œuvre de Wisner», Travailler. Revue internationale de psychopathologie et psychodynamique du travail, pp. 207-218.

DidRY C. (2002), Naissance de la convention collective. Débats juridiques et luttes sociales en France au début $d u X X^{e}$ siècle, Paris, éditions de l'École des hautes études en sciences sociales.

Duraffourg J., Vuillon B. (sous la direction de) (2004), Alain Wisner et les tâches du présent. La bataille du travail réel, Toulouse, Octarès.

Halbwachs M. (1912), L’Année sociologique, 19091912, t. 12, pp. 757-758.

Haughton S. (1873), Principles of animal mechanics, Londres.

Huteau M., Lautrey J. (1979), «Les origines et la naissance du mouvement de l'orientation », L'Orientation scolaire et professionnelle, vol. 8, $\mathrm{n}^{\circ} 1$, pp. 3-43.

IMBERT A. (1880), Recherches théoriques et expérimentales sur l'élasticité du caoutchouc, thèse de sciences, Lyon.

IMBERT A. (1883), De l'interprétation et de l'emploi du pouvoir dioptrique et de la dioptrie métrique en ophtalmologie, thèse de médecine, Lyon.

Imbert A. (1887), «Les anomalies de la vision: notions élémentaires", extrait de la Gazette hebdomadaire des sciences médicales de Montpellier, conférence aux élèves de l’école normale de Montpellier.

Imbert A. (1902a), «Un cas d'expertise. Fracture de l'avant-bras », Annales d'hygiène publique et de médecine légale, série 3, n 47, pp. 385-398.

IMBERT A. (1902b), «Mode de fonctionnement économique de l’organisme», Scientia, série biologique, $\mathrm{n}^{\circ} 14$, p. 9.

ImBert A. (1904), «Les accidents du travail et les compagnies d'assurances", Revue scientifique, $\mathrm{n}^{\circ} 23$ (tome I), pp. 711-719.

Imbert A. (1905), « Maladies professionnelles », Moniteur des syndicats ouvriers. Organe des chambres syndicales ouvrières, 3 septembre.
IMBERT A. (1906), «Les revendications ouvrières et la science expérimentale», Revue scientifique, $\mathrm{n}^{\circ} 17$ (tome V), pp. 521-524: 522.

IMBERT A. (1907), «L'étude scientifique expérimentale du travail professionnel », L'Année psychologique, pp. 245259.

ImBert A. (1908a), «Syndicats ouvriers et compagnies d’assurance », Grande Revue, pp. 97-122: 120.

IMBERT A. (1908b), «Le surmenage par suite du travail professionnel » (compte rendu du IV ${ }^{\mathrm{e}}$ congrès international d'hygiène et de démographie, Berlin, septembre 1907), Année psychologique, 1908, pp. 232-248: 247.

IMBERT A. (1909a), "Rôle des ouvriers dans certains congrès scientifiques», Grande Revue, pp. 574-578: 575.

IMBERT A. (1909b), «Étude expérimentale du transport de charges avec une brouette», Bulletin de l'inspection $d u$ travail, $\mathrm{n}^{\circ} 1-2$, p. 911.

IMBERT A (1909c), «Les expériences physiologiques relatives à la dépense de force dans le travail », Quatrième congrès de l'hygiène et de la sécurité des travailleurs et des ateliers, Paris, L'émancipatrice, imprimerie communiste, p. 75.

IMBERT A (1909d), Rôle de la physiologie dans les conflits entre le capital et le travail, Paris, Imprimerie nationale.

IMBERT A. (1910), «Évaluation de la capacité de travail avant et après un accident du travail », Revue scientifique, p. 42.

IMBERT A. (1911a), Observations économiques de vie ouvrières, Paris, Masson.

Imbert A. (1911b), «Les méthodes du laboratoire appliquées à l'étude directe et pratique des questions ouvrières ", Revue générale des sciences pures et appliquées, vol. 12, pp. 478-486: 482.

IMBERT A. (1912), «Un nouveau champ d'action en hygiène sociale. L'étude expérimentale du travail professionnel », Bulletin de l'Alliance d'hygiène sociale, p. 3584.

IMBERT A. (1913a) «Tableau méthodique des professions qui appellent des recherches, et nature de ces recherches », AN F22/526.

Imbert A. (1913b), Accidents du travail. Guide pour l'évaluation des incapacités, Paris, Masson et Cie.

IMBERT A. (1915), Rapport du service central de physicothérapie militaire de la $16^{e}$ région, Imprimerie générale du Midi, Montpellier.

IMBERT A. (1920), Le système Taylor. Analyse et commentaires, Paris, Ravisse. 
IMBERT A. (1922), « Mode de fonctionnement économique du cerveau », Année psychologique, pp. 221-223.

Imbert A. (1929), "Transport de charges avec une charrette à bras ", Revue de la science du travail, $\mathrm{n}^{\circ} 1$, pp. 197-216.

Imbert A., Mestre A. (1904), «À propos de l'influence de la fatigue professionnelle sur la production des accidents du travail », Revue industrielle, 5 novembre, $n^{\circ} 45$, pp. 449-450: 450.

Imbert A., Mestre A. (1905a), «Nouvelles statistiques d'accidents du travail», Revue scientifique, 17, p. 530.

Imbert A., Mestre A. (1905b), «Recherche sur la manœuvre du cabrouet et la fatigue qu'il en résulte», Bulletin de l'inspection du travail, pp. 374-406.

ImBert Léon (1913), Accidents du travail. Guide pour l'évaluation des incapacités, Paris, Masson et Cie.

IMBERT Lucien (1996), «Armand Imbert (1850-1922), sa vie, son œuvre», Nunc Monspeliensis Hippocrates, pp. 17-22.

LAHY J.-M. (1916), Le système Taylor et la physiologie du travail professionnel, Paris, Masson.

Moret-Lespinet I. (1997), Théories et pratiques républicaines de la réforme sociale: l'Office du travail 18911914, thèse de $3^{\mathrm{e}}$ cycle, université Paris X.

Moutet A. (2004), «La psychologie appliquée à l'industrie: un moyen de définition de l'aptitude ou de l'inaptitude »? (de 1930 à 1960), in Catherine Omnès et Anne-Sophie Bruno, Les mains inutiles. Inaptitude au travail et emploi en Europe, Paris, Belin, pp. 83-101.

Omnès C. (2002), «Les premiers pas des services du personnel. La gestion du facteur humain dans les entreprises européennes des années 1920 », Histoire et sociétés. Revue européenne d'histoire sociale, $\mathrm{n}^{\circ} 2$.

Pons F. (1912), Les accidents du travail. Commentaire pratique, Marseille, Conseil judiciaire de la Bourse du travail (CGT).
Rabinbach A. (2004), Le moteur humain. L'énergie, la fatigue et les origines de la modernité, Paris, La Fabrique (édition originale 1990).

RASMussen A. (2001), «L'hygiène en congrès (18521912): circulation et configurations internationales ", in Patrice Bourdais (ed.), Les Hygiénistes. Enjeux, modèles et pratiques (XVIII ${ }^{e}-X I X^{e}$ siècles), Paris, Belin.

Ribeill G. (1980), «Les débuts de l’ergonomie en France à la veille de la Première Guerre mondiale », Le mouvement social, $n^{\circ} 11$, octobre-décembre, pp. 3-36.

Ribeill G. (1986), «L'organisation physiologique du travail », Cadres CFDT, n 324, pp. 50-58.

Rотн E., (1907), «Ermüdung durch Berufsarbeit», Comptes rendus du congrès international d'hygiène et de démographie, Berlin, p. 618.

Soubiran-Paillet F. (2007), «Parlement, administrateurs et experts. Le discours de la compétence», Vingtième siècle, pp. 151-163.

Turbiaux M. (2003), «La résistible création d'un laboratoire du travail», communication orale, Comité pour la célébration du centenaire du ministère du Travail, CEDIAS/Musée social, Paris, 13 novembre.

VATin F. (1999), Le travail. Sciences et société, Bruxelles, éditions de l’Université de Bruxelles.

VATin F. (2006), «Ergonomie-Ergologie», Bulletin de la Société française pour l'histoire des sciences de l'homme, n² 29, été, pp. 52-62.

VIET V. (1994), Les voltigeurs de la République: l'inspection du travail en France jusqu'en 1914, Paris, éditions du CNRS.

Viet V. Ruffat M. (1999), Le choix de la prévention, Paris, Economica, 1999.

Weber M. (1995), Zur Psychophysik der industriellen Arbeit, Tübingen, Mohr (Siebeck).

Wundt W. (1884), Traité élémentaire de physique médicale, Paris, Baillière (1 $1^{\text {re }}$ édition allemande 1867). 
